\title{
Experimental and Clinical Evidence Based Rationality of Incorporation of Deep Fascia in Tissue Transfer for Reconstructive Surgery
}

\author{
V. Bhattacharya \\ Former HOD, Plastic Surgery, Institute of Medical Sciences, \\ Banaras Hindu University, Varanasi, India.
}

\begin{abstract}
Intense clinical research since 1984 through 93 parameters has unveiled the rationality of reconstructive procedures with several new concepts and innovative techniques of various compositions. Such extensive work has been done totally in our country popularizing the techniques amongst the plastic surgeons of the world. This presentation deals with the tissue constituents and vascular network of the deep fascia. This sustained research was conducted at inter-departmental, interfaculty and interinstitutional levels.
\end{abstract}

Fresh cadaveric dissections, animal experimentations and clinical research revealed crucial findings applicable for resurfacing defects of different etiology and magnitude. The deep fascia covering the muscle is thought be an inert a avascular structure with protective function only. We have demonstrated for the first time in the world the live vascular and lymphatic microcirculation in deep fascia proving it to be having dense vascular network. Therefore its incorporation during the reconstructive surgery enhances the vascularity allowing transfer of large dimension of tissue for reconstruction in cases of trauma, infection, cancer surgery, etc. It has proved the rationality of these procedures convincingly to the scientific world.

Histology of deep fascia showed rich subfascial and suprafascial arterioles and capillaries. Intrafascial course of the perforating vessels from the subfascial plane to the suprafascial plane was visualized. Confocal microscopic analysis of fluorecenised deep fascia under 40X magnification showed longitudinally aligned collagen fibres and nuclei of multiple fibroblasts. Electron microscopic Y of deep fascia revealed (a) Elastic tissue and collagen fibres, (b) Lymphatic vessel, (c) Thin walled venule with

Correspondence : Dr. V. Bhattacharya, B-33/14-16, Gandhi Nagar, Naria, Varanasi221005 (UP).

COL. SANGHAM LAL MEMORIAL ORATION delivered during NAMSCON 2016 at the All India Institute of Medical Sciences, Raipur. 
single layer of muscle fiber, endothelial cell nucleus, Venules with multiple RBCs, mast cell with granules and capillary showing endothelium and endothelial cells.

Angiography in experimental model and patients, demonstrated longitudinally oriented vascular network in deep fascia and fasciocutaneous flap. Live microcirculation and Lymphatic circulation in the human deep fascia was demonstrated first time in the world under 150 and 600 magnifications.

Keywords : Deep Fascia, reconstructive Surgery, Microcirculation.

INTRODUCTION

Frequently resurfacing of defects is required following trauma, excision of malignant lesion, avulsion injuries, chronic wounds, etc. Lower limb is often involved. The composite soft tissue commonly used for reconstruction consists of skin, subcutaneous tissue, deep fascia and/or muscle (1). The vascular supply exists in all these tissue planes in the form of dense vascular arcade. In the lower limb, there are three main vascular trunks namely, posterior tibial, peroneal and anterior tibial. Small vessels arise from them which perforate different tissue planes and are called 'perforators'. There are musculocutaneous, septocutaneous and direct cutaneous perforators (2). As they pass through different planes, dense vascular networks are formed which communicate with each other (3). Thus a continuous flow of blood is ensured in the composite tissue transfer by incorporating the perforators and the vascular network of tissues (4). In this context the deep fascia covering the muscles plays an important role. It is a highly vascular structure contrary to the common belief. It has a suprafascial and a subfascial vascular plexuses.
Therefore to ensure circulation, knowledge of two aspects is important.

(1) Main vascular trunks and perforators.

(2) Vascular network in the tissues.

Why it is necessary? It explains in clinical practice how large dimensions of tissue are successfully transferred based on only these perforators and the vascular arcades (5). Hence to understand the dynamics of circulation, we need to know about supplying vessels and the microcirculation in the tissues. Knowledge of perforators deals with location, number, caliber, direction, and the perfusion pressure. Microcirculation includes two systems, vascular and lymphatic. To convince the scientific world, evidence based proof is necessary regarding the above features. This presentation distinctly emphasizes the rationality of the success of these reconstructive procedures. There are different methods to visualize the perforators. They are :
(a) Histology of deep fascia
(b) Old and fresh cadaveric dissections
(c) Audio Doppler
(d) Color Doppler 
(e) CT angiography with $3 \mathrm{D}$ reconstruction. All these findings establishing the practical utility in reconstructive surgery has been conducted in our institute through collaboration of different departments. Such research requires original thinking and formulation of experimental and clinical model.

Histology of deep fascia and cadaveric dissection:

The knowledge regarding the structural details of deep fascia remains inadequate. It was described to be relatively avascular covering the muscles

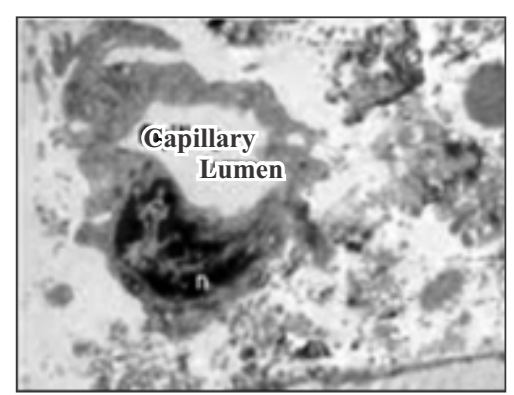

Fig. 1(a)

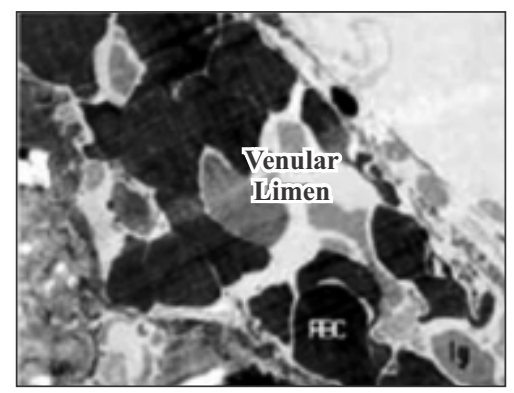

Fig. 1(c) having predominantly protective function. Some studies revealed that it has associated vascular arcade and hence its incorporation ascertained additional vascularity to the flaps. Light microscopy $(10 X \& 40 X)$ of fresh human deep fascia harvested from the covering of the gastrocnemeus muscle was done. It showed arteriols and capillaries in both the layers of deep fascia (suprafascial and subfascial plexes). They could be seen traversing the intrafascial course. Under electron microscopy (4000X), arteries, capillaries, venules and lymphatics could be seen apart from other structural elements (6) (Figs. 1a, b, c, d). They all contribute to enhance the vascularity and

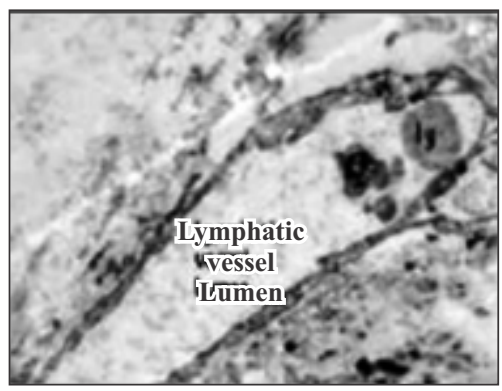

Fig. 1(b)

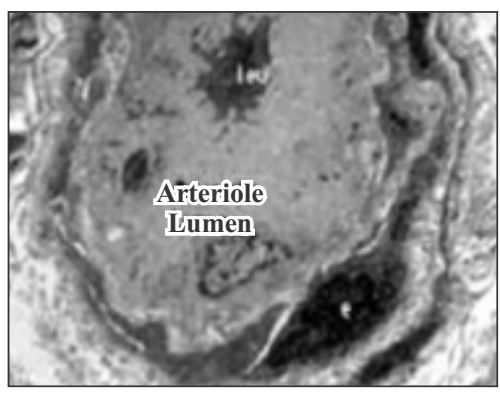

Fig. 1(d)

Fig. 1 : Electron microphotograph of the deep fascia showing (A) Capillary, the single endothelial cell nucleus (n) (x4000). (B) Lymphatic vessel with lymphocyte in the lumen (ly) (x4000). (C) Venule with lymphocyte (ly) and RBCs in the lumen (x 4000). (D) Arteriole with leukocyte (leu) in the lumen (x4000). 
maintain the physiological functions of the transferred tissue.

Dissection on old cadavers in the lower limb revealed presence of perforators of different sizes, arising from the main vascular trunks, at regular intervals. Similar findings were confirmed in fresh cadaveric dissection after injecting dye in the poplitial artery (7) (Figs. 2 \& 3). During operative procedure

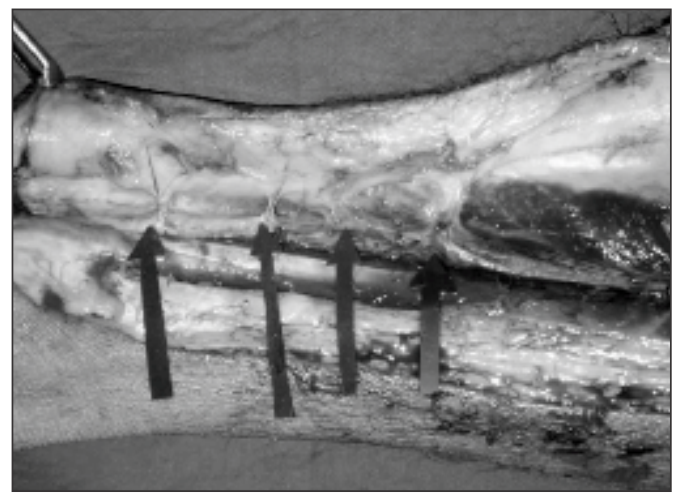

Fig. 2 : Cadaveric dissection showing distal posterior tibial perforators.

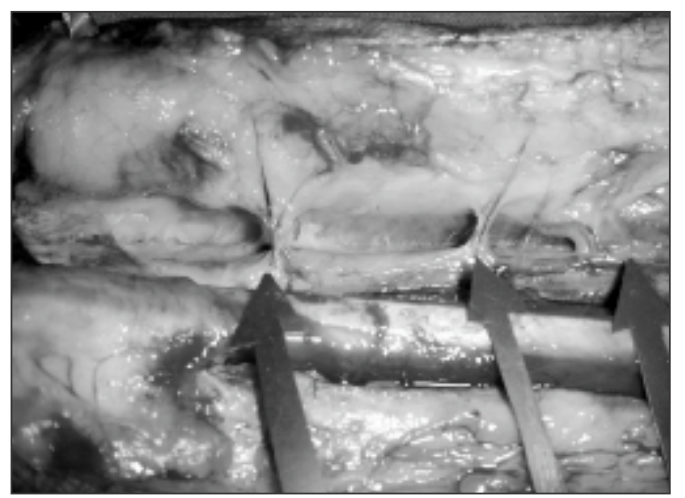

Fig. 3 : Fresh cadaveric dissection showing perforators with their vena comitantes, either branching prior to reaching the fascia or continuing without branching.

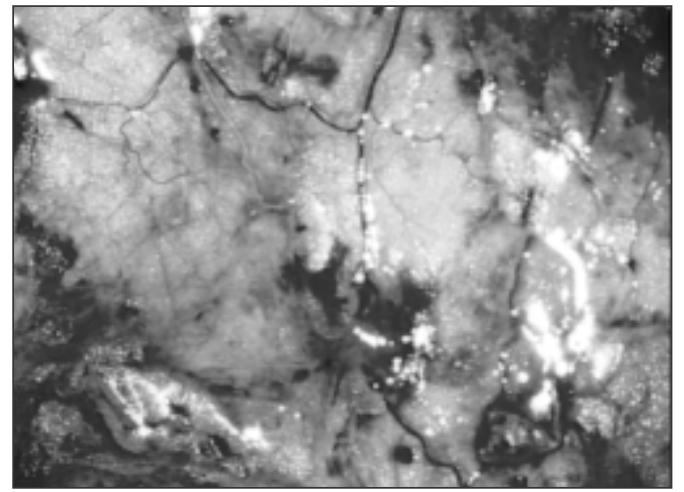

Fig. 4 : Intraoperative view under magnification showing the intrafascial course of the perforator merging with the subfascial plexus.

of flap elevation, exploratory incision also confirms the location of these perforators. Intraoperatively under magnification the intrafascial course of the perforator merging with the subfascial plexus can be witnessed (Fig. 4).

\section{Doppler studies :}

Color Doppler provides important information regarding the location, direction, length and the internal diameter of the perforators. In clinical practice, the Audio Doppler can locate them and provides idea about their dimension depending upon the intensity of sound.

\section{CT Angiography:}

The evolution of flaps has come a long way in terms of tissue constituents and mode of transfer. This advancement could be possible due to better delineation and understanding of the vascular system and its ability to perfuse a flap (8). The locoregional perforator flaps are preferred 
for reconstruction whenever adjacent normal tissue of required dimension is available. The two most important reasons are that :

(a) It does not sacrifice any major vessel in an already traumatized limb and

(b) It does not require microvascular free tissue transfer. However, a free flap from a distant area can also be planned to be anastomosed to a perforator. Thus the knowledge of perforators is of significant value whether one chooses to apply a locoregional flap or a free flap depending upon the situation. The perforator flaps evolved on the knowledge of the vascular tree from the main vascular trunk up to the subdermal plexus. Therefore, we thought that it was necessary to map

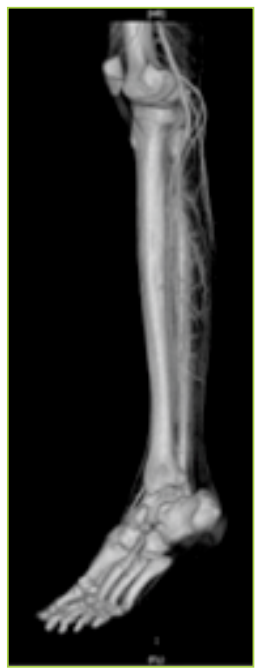

Fig. 5 : CT angiography showing the perforators of the posterior tibial artery. the whole vascular arcade in the lower limb.

To evaluate the perforators and the whole vascular tree, peripheral CT angiography with $3 \mathrm{D}$ reconstruction was done. It helps in designing flaps of different constituents based on the selected perforators. CT angiography was done using a non-ionic iodinated contrast media injected through the antecubital vein. The lower limbs were imaged using volume rendering $\mathrm{CT}$ scan machine. Three dimensional reconstructions were made. The whole arterial tree, along with the perforators, was mapped. The three dimensional CT angiographic reconstruction with bone and soft tissue provided advanced knowledge of this vascular network (9) (Figs. 5 \& 6). It delineated the main vessels, the perforators, their caliber, inter -perforator

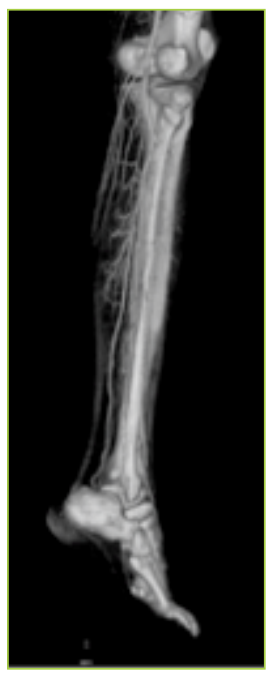

Fig. 6 : CT angiography showing the perforators of the peroneal artery. 


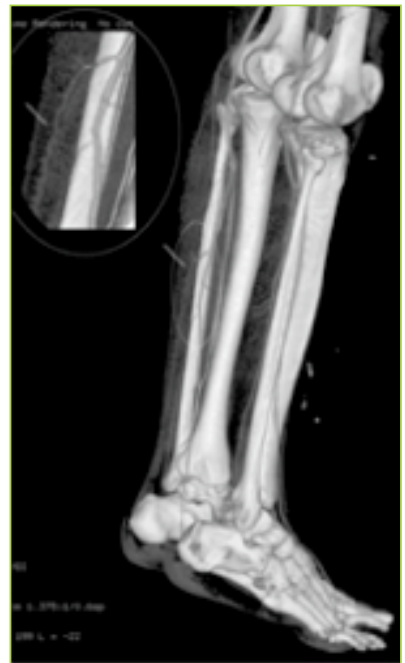

Fig. 7 : (a) Course of the perforators up to the subdermal plexus of the posterior and peroneal arteries (b) The inset showing the magnified view of the course of the peroneal perforator.

distance, direction, distance from fixed bony landmarks and course up to the subdermal plexus (Fig.7).

By 360 degree rotation and dynamic 3D CT reconstruction, every detail of the vascular tree could be delineated which otherwise will be missing in a single slice. This is an advancement over the existing two dimensional sagittal tomography which does not provide the detailed findings required for reconstruction. The intra-operative findings of perforators during flap dissection confirmed the accuracy of peripheral CT angiography.

\section{Perfusion pressure:}

We correlated between the internal diameters of perforators with their perfusion pressure. This is an important step towards understanding how much tissue is supplied by a perforator. It is difficult to assess the angiosome of the perforators. However it is well established now that due to the rich inherent vascular network of the deep fascia, one can dissect a large flap of nonconventional dimension (beyond 1:2) safely and it behaves like an axial flap. This is because the vascular networks of adjacent perforators freely anastomose with each other and make an effective continuous vascular channel. The standardization was done in the experimental model in rats in the department of physiology. Prior to measurement, color Doppler gave the information regarding the location and the internal diameter of the perforator to be cannulated. During dissection of the flaps, we anyway divide the proximal perforators. One of such perforator was identified under loupe magnification and skeletonized for the study. Therefore no harm was done to the patient. The artery was separated from the venae commitantes. The P-50 polyethylene microcannula was passed gently into the proximal loop of the artery. Free flow of arterial blood through the cannula confirmed the proper cannulation. The blood sample was taken for measurement of arterial blood gases so as to confirm the placement of the cannula in an artery rather than a vein. The cannula was then connected to the precalibrated physiograph, which was used in the experimental model. Recordings were then taken which were calculated by the equations as mentioned for the different sensitivities, in experimental model (10). 
Thus we measured the perfusion pressure in a perforator of known internal diameter and found that they were directly proportional to the internal diameter. The perforators were classified depending upon the internal diameter as small (1.2 $\mathrm{mm}$ or less), medium (1.3-2.0 $\mathrm{mm})$ and large (more than $2.0 \mathrm{~mm}$ ). In small, medium and large sized perforators the range of perfusion pressure was 20-25, 20-33 and 32-49 $\mathrm{mmHg}$, respectively.

\section{Microcirculation :}

Anatomy of text books do not stress the importance of the vascularity of the deep fascia except for its structural constituents and functional ability to cover the muscles. Histologic study proved the existence of subfascial and suprafascial network.

The rich vascular network in the deep fascia have been emphasized by various scientists, but the actual demonstration of live circulation in the deep fascia has not been previously witnessed. Encouraged by the sight of live circulation in the web membrane of toad hind limb in experimental model, a successful attempt was made to demonstrate the live circulation in the vascular network of the human deep fascia. Fascial extension of inferiorly based fasciocutaneous flaps were dissected in patients with distal leg and heel defects (11). The fascial extension in continuity with a proximal retrograde fasciocutaneous flap was mounted on a glass slide under natural tension and examined under a microscope under $150 \mathrm{X}$ and 600X magnification (Fig.8). The anastomotic channels and circulation could be seen clearly in both layers. We witnessed live microcirculation and the movement of individual red blood corpuscles in the vascular channels of the deep fascia (Figs. 9a \& b). In a field of observation, there were single or multiple vessels, vascular wall was well delineated, blood was flowing in a high speed, individual cells could be seen moving. We also noticed that the deep fascia has two layers with circulations that are independent of each other. A video recording was done to document these important features. This study led us to understand the intensity and magnitude of microcirculation associated with the deep fascia and to prove the rationale for incorporating the deep fascia to provide safety to the flap. This also explains why flap of large dimensions can be transferred

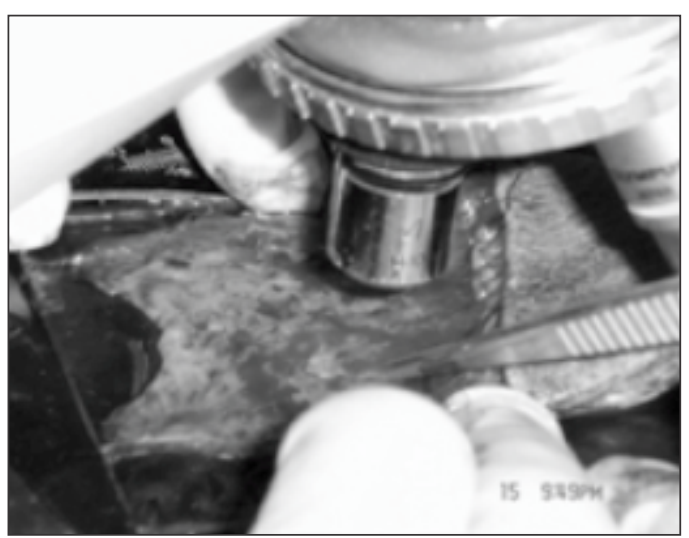

Fig. 8 : The fascial extension in continuity with a proximal retrograde fasciocutaneous flap was mounted on a glass slide under natural tension and examined under a microscope under $150 X \& 600 X$ magnifications. 


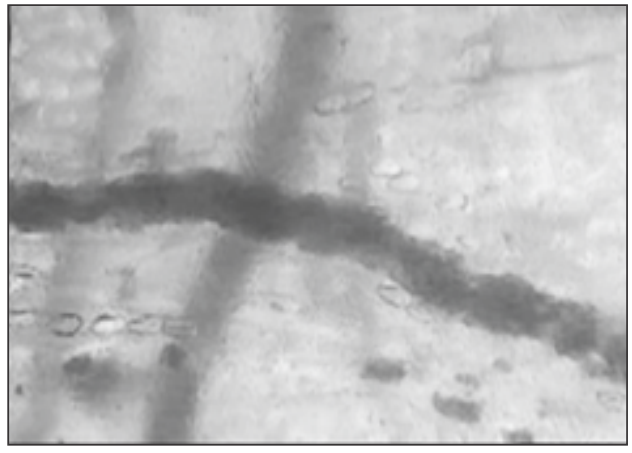

Fig. 9 (a) :

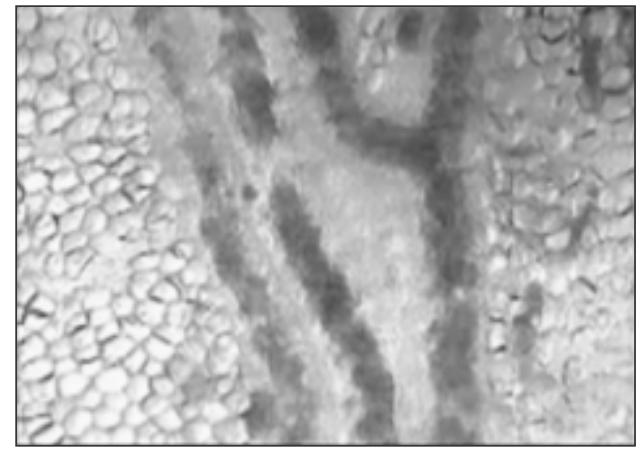

Fig. 9 (b) :

a\&b- Photomicrographs showing multiple vascular channels in the deep fascia.

for reconstruction of defects exposing underlying vital structures. Such vascular network exists in every tissue of our body with blood flow. However we cannot demonstrate live circulation in them as they are thick tissue and demonstration of circulation is only possible in transparent tissue which can be observed under the lance of the microscope.

\section{Lymphatic circulation:}

It is well known that vascular arcade and lymphatic network co-exist all over the body with distinct physiological

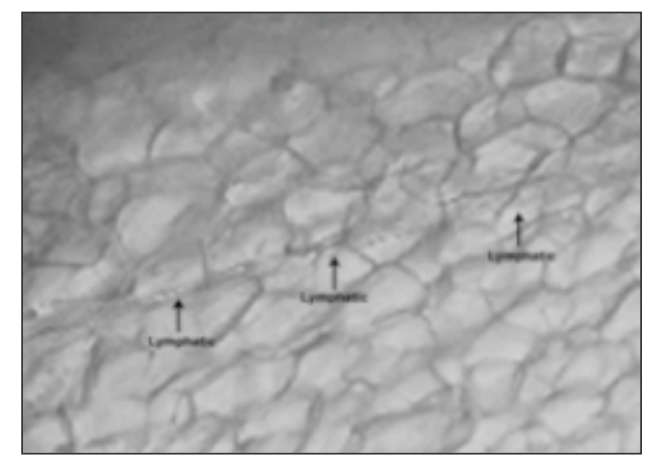

Fig.10 : Thin walled lymphatics containing straw colored lymph with suspended large molecules of fat and protein. functions complimentary to each other. We visualized lymphatics in the deep fascia under electron microscope with 4000 magnification. Encouraged by our previous research of demonstration of live microcirculation in the deep fascia, we successfully explored the possibility of demonstrating live lymphatic circulation. Using the same clinical model, we examined under microscope using 600 magnifications (12). We witnessed live microcirculation as well as live lymphatic circulation in the same field of observation with specific characteristics (Figs. $10 \& 11$ ). A video recording was made to document these important

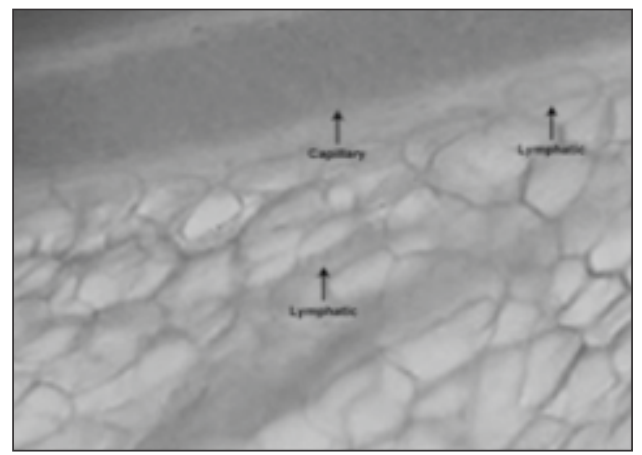

Fig.11: The caliber of the vessel is

20-25 times more than the lymphatic caliber. 
features which to the best of our knowledge is not mentioned in the literature. The rich vascular network in the deep fascia can be easily witnessed by naked eye when ever any surface (supra or subfascial) of the deep fascia is observed during various surgical procedures. However the lymphatic system cannot be seen in routine histology or by naked eye. It was an exciting experience for the entire team to witness vibrant live microcirculation and lymphatic circulation in the deep fascia. Microphotography and video recording were performed. It was interesting to compare the live lymphatic circulation and live microcirculation in the same field of observation.

There were several interesting findings:

(1) In the same field the circulation in blood vessels (capillaries and arterioles) and lymphatics could be seen.

(2) The caliber of the vessels were 2025 times more than the lymphatic.

(3) The wall of the lymphatics were thinner.

(4) The content of lymphatic was light straw colored with suspended large molecules of fat and protein (lymph) as compared to the red content of blood in the vessels.

(5) The rate of flow of lymph was much slower in lymphatics than rapid blood flow in the vessels.

(6) Depending upon the network, the flow in the lymphatics could be in different directions in the multiple channels in the same field.

(7) At places the lymphatic crossed over a vessel proving it to be a different system.
(8) Due to the variation of thickness of the wall of vessels and lymphatics, it was difficult to distinctly focus both the systems simultaneously at 600 magnification.

(9) In still photography the lymphatics almost simulate the septas separating the fat globules making it difficult to distinguish them. However the lymphatics are continuous channels and septas are around fat globules separating them having segmental appearance.

(10) Therefore only live lymphatic circulation can precisely identify these features.

Based on these informations emerged the supporting role of the lymphatics in tissue transfer. The gradual reduction in edema of the flap can be correlated with the lymphatic circulation as one of the major component. This further proves that inclusion of deep fascia in a flap has immense beneficiary effect.

\section{Conclusion :}

The successful tissue transfer in reconstructive surgery is based on strong rationality. The dynamics of circulation has two parts

(a) The supplying vessels (perforators) and

(b) The dense vascular network in different tissue planes. This presentation deals in detail about the perforators arising from the main vessels and their visualization through several clinical parameters. Through original research it also establishes, the vascular network 
associated with the deep fascia and the live microvascular and microlymphatic microcirculation in it. We could also establish that the deep fascia consists of two layers which have individual vascular network and circulation. All these information are of immense value in the field of reconstructive surgery.

\section{REFERENCES :}

1. McCarthy (1990). Plastic Surgery. General Principles. Vol. 1, Philadelphia: WB Saunders Company, 275-377.

2. Ponten B (1981). The fasciocutaneous flap: its use in soft tissue defects of lower leg. BJPS 34: 215220.

3. Taylor GI, Palmer JH (1987). The vascular territories (angiosomes) of the body: Experimental study and clinical applications. BJPS 40: 113141.

4. Bhattacharya V, Reddy GR, Goyal S, Kumar U (2007). Skeletonised retrograde distal perforator island fasciocutaneous flaps for leg and foot defects. J Plast Reconstr Aesthet Surg 60: 892-897.

5. Cormack GG, Lamberty BGH. The fasciocutaneous system of vessels. The Arterial Anatomy of Skin Flap. Churchill Livingstone, 1986.

6. Bhattacharya V, Barooah PS, Nag $\mathrm{TC}$, et al. (2010). Detail microscopic analysis of deep fascia of lower limb and its surgical implication. IJPS 43: 135-140.

7. Bhattacharya V, Goyal S (2006). A dye study in fresh cadavers to outline retrograde flaps of the lower limb and to examine clinical implications. European Journal of Plastic Surgery 28:458-463.

8. Bhattachatya V, Goyal S, Jain P, Singh NS (2006). Angiographic evaluation of fasciocutaneous flaps. Int Surgery 91: 326-331.

9. Bhattacharya V, Agrawal NK, Chaudhury GR, et al. (2012). CT Angiographic evaluation of perforators in the lower limb and their reconstructive implication. IJPS 45: 494-497.

10. Bhattacharya V, Deshpande SB, Watts RK, et al. (2005). Measurement of perfusion pressure of perforators and its correlation with their internal diameter. BJPS 58:759-764.

11. Bhattacharya V, Watts RK, Reddy GR (2005). Live demonstration of microcirculation in the deep fascia and its implication. Plast Reconstr Surg 115: 458-463.

12. Bhattacharya V, Choudhuri GR, Mishra B, Kumar U (2011). Demonstration of live lymphatic circulation in the deep fascia and its implication. European Journal of Plastic Surgery 34(2): 99-102. 